\title{
DESIGN AND ANALYSIS OF CONDENSER USING 3D MODELING SOFTWARE
}

\author{
A. Harsha Vardhan Reddy ${ }^{1}$, G.Rajasekhar Reddy ${ }^{2}$, G.Phanindra ${ }^{3}$, K. Vijay Kumar ${ }^{4}$ \\ ${ }^{1}$ Asst. Professor, Mechanical, Gurunanak Institutions, Telangana, India \\ ${ }^{2}$ Student, mechanical, Gurunanak Institutions, Telangana, India \\ ${ }^{3}$ Student, mechanical, Gurunanak Institutions, Telangana, India \\ ${ }^{4}$ Student, mechanical, Gurunanak Institutions, Telangana, India
}

\begin{abstract}
Refrigeration systems have condenser that removes unwanted heat from the refrigerant and transfers that heat outdoors. The primary component of a condenser is typically the condenser coil, through which the refrigerant flows. Since, the condenser coil contains refrigerant that absorbs heat from the surrounding air, the refrigerant temperature must be higher than the air. In this thesis heat transfer by convection in refrigeration by varying the condenser length are determined by CFD and thermal analysis. The assessment is out on an air-cooled tube condenser of a vapour compression cycle for refrigeration system. The materials considered for tubes are Copper and Aluminum alloys. The refrigerants varied will be $R$ 12. CFD analysis is done to determine temperature distribution and heat transfer rates by varying the refrigerants. Heat transfer analysis is done on the condenser to evaluate the better material. 3D modeling is done in CREO and analysis is done in ANSYS.
\end{abstract}

Keywords: CREO, ANSYS, CFD, FEA

\section{INTRODUCTION}

In systems involving heat transfer, a condenser is a device or unit used to condense a substance from its gaseous to its liquid state, by cooling it. In so doing, the latent heat is given up by the substance, and will transfer to the condenser coolant. Condensers can be made according to numerous designs, and come in many sizes ranging from rather small (hand-held) to very large (industrial-scale units used in plant processes). For example, a refrigerator uses a condenser to get rid of heat extracted from the interior of the unit to the outside air. Condensers are used in air conditioning, industrial chemical processes such as distillation, steam power plants and other heat-exchange systems. Use of cooling water or surrounding air as the coolant is common in many condensers

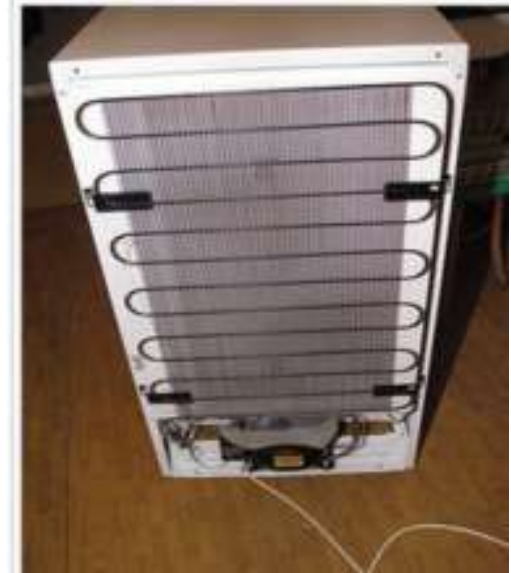

Fig 1: Condenser of refrigerator

\subsection{Types of Condensers}

- Direct contact condenser

- Water-cooled

- Air-cooled

- Evaporative

\subsection{Block Diagram of a Condencer}

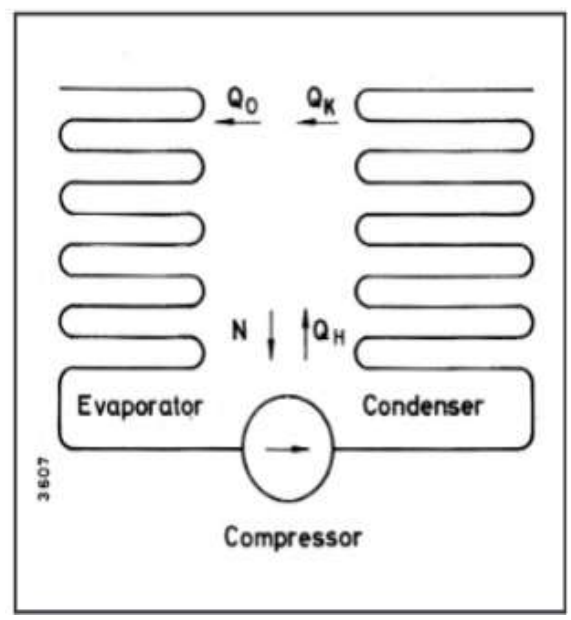

Fig 2: Block diagram

\section{LITERATURE REVIEW}

A comprehensive review of the literature on Vapor Absorption Systems, Compression-Absorption System and Vapor Compression System has been carried out on various aspects of energy analysis, the type of cycles analyzed, working pairs used and energy analysis. With regards to 
vapor absorption cycles, it is found that mostly the studies are carried out on large capacity systems and the investigation had been carried out with in a limited range of system design parameters. The literature on small vapor absorption systems is scant and very few studies have been done on smaller systems. The above studies are simulation studies. Regarding compression-absorption systems studies have been carried out by many researchers mostly analytically and experimentally. The investigations have been done on wet compression cycles which eliminated the need of solution pump. The literature provides details with regard to the applications.

\section{INTRODUCTION TO CREO}

PTC CREO, formerly known as Pro/ENGINEER, is 3D modeling software used in mechanical engineering, design, manufacturing, and in $\mathrm{CAD}$ drafting service firms. It was one of the first 3D CAD modeling applications that used a rule-based parametric system. Using parameters, dimensions and features to capture the behavior of the product, it can optimize the development product as well as the design itself.

\section{D Model}

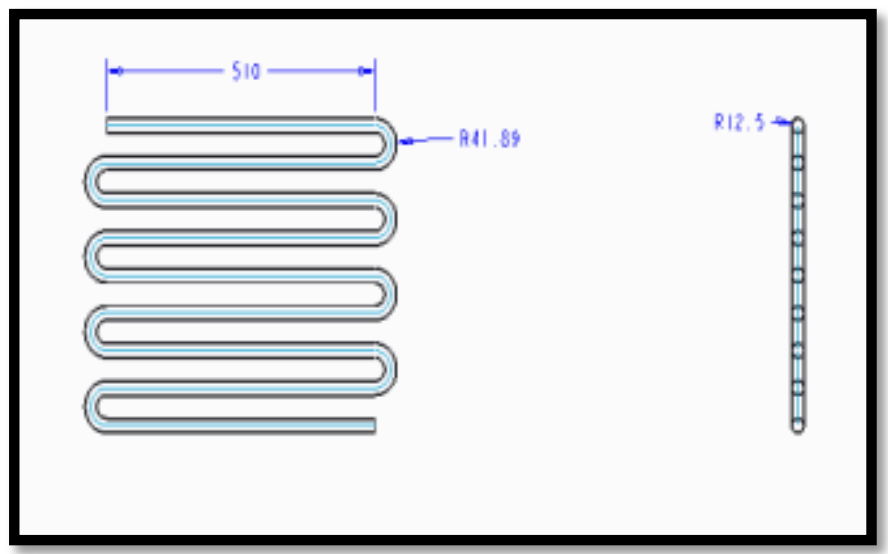

Fig 3: 3D MODEL

\section{INTRODUCTION TO ANSYS}

\section{Structural Analysis}

ANSYS Autodyn is computer simulation tool for simulating the response of materials to short duration severe loadings from impact, high pressure or explosions.

\section{ANSYS Mechanical}

ANSYS Mechanical is a finite element analysis tool for structural analysis, including linear, nonlinear and dynamic studies. This computer simulation product provides finite elements to model behavior, and supports material models and equation solvers for a wide range of mechanical design problems. ANSYS Mechanical also includes thermal analysis and coupled-physics capabilities involving acoustics, piezoelectric, thermal-structural and thermoelectric analysis.

\section{Fluid Dynamics}

ANSYS Fluent, CFD, CFX, FENSAP-ICE and related software are Computational Fluid Dynamics software tools used by engineers for design and analysis.

\subsection{Thermal Analysis of Condenser}

\subsubsection{Condenser Length-345mm}

Material-Aluminum Alloy

Imported Model

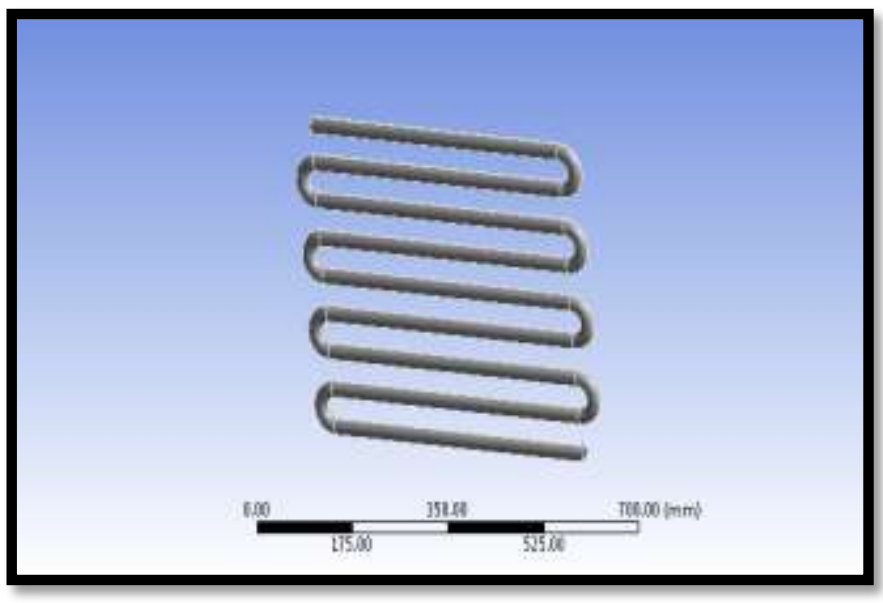

Fig 5: Imported Model

\section{Meshed Model}

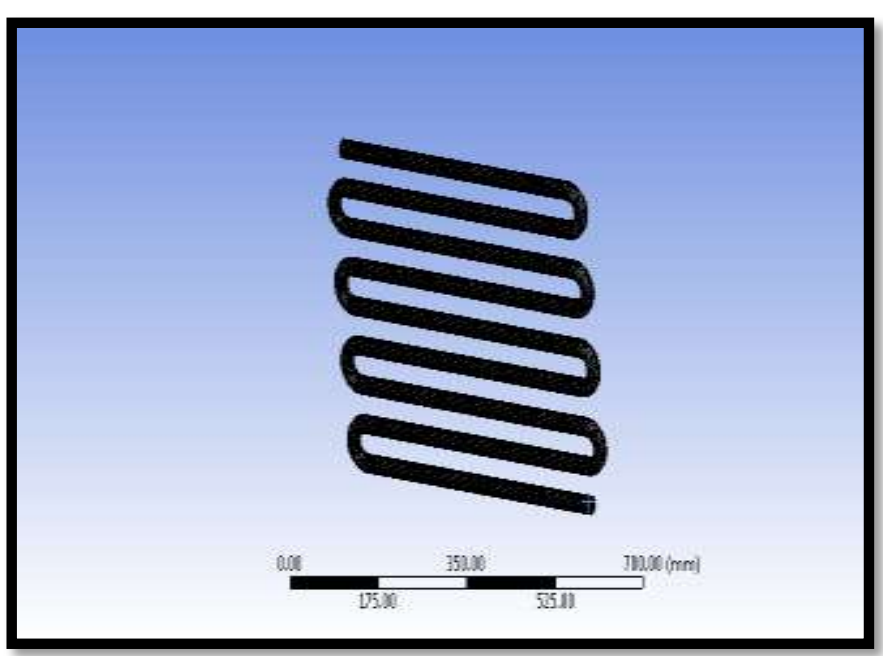

Fig 6: Meshed Model

Finite element analysis or FEA representing a real project as a "mesh" a series of small, regularly shaped tetrahedron connected elements, as shown in the above fig.

\section{BOUNDARY CONDITIONS}

$\mathrm{T}=278 \mathrm{~K}$

\section{Temperature}




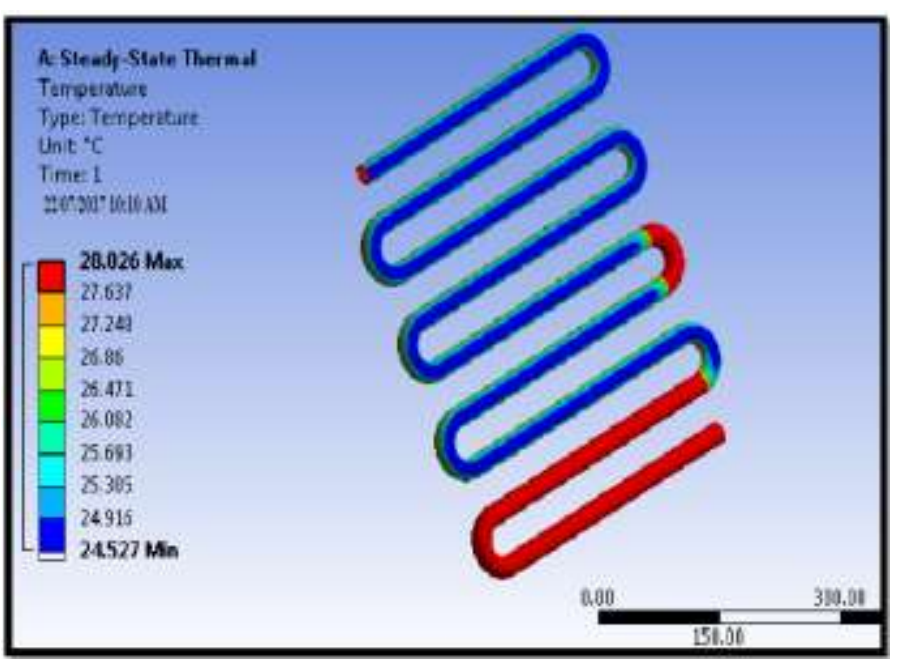

Fig 7: TEMPERATURE

\section{Heat Flux}

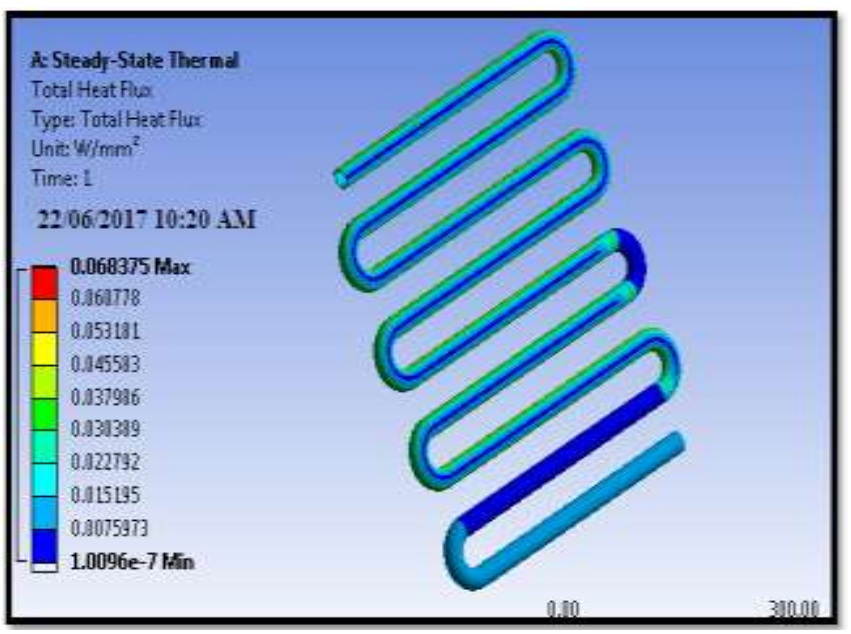

Fig 8: HEAT FLUX

\section{Material -Copper}

\section{Temperature}

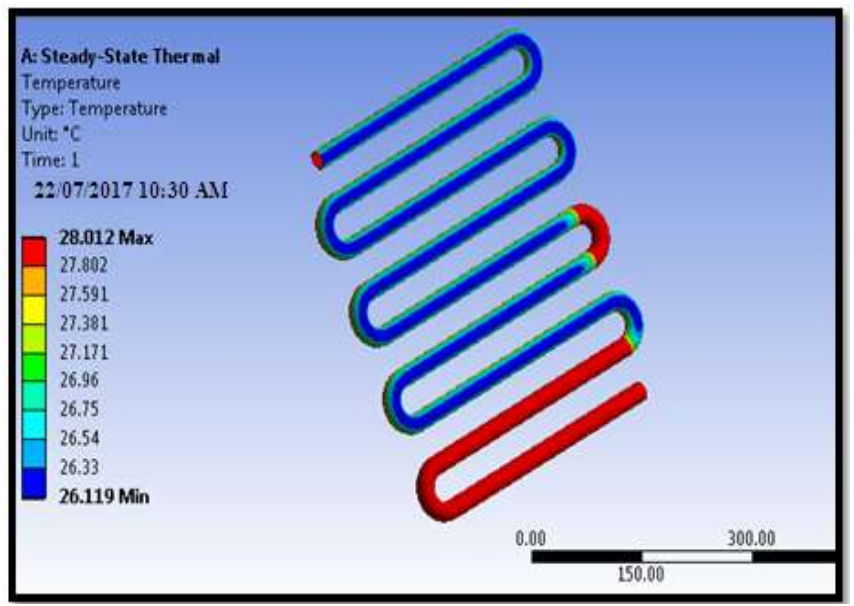

Heat Flux

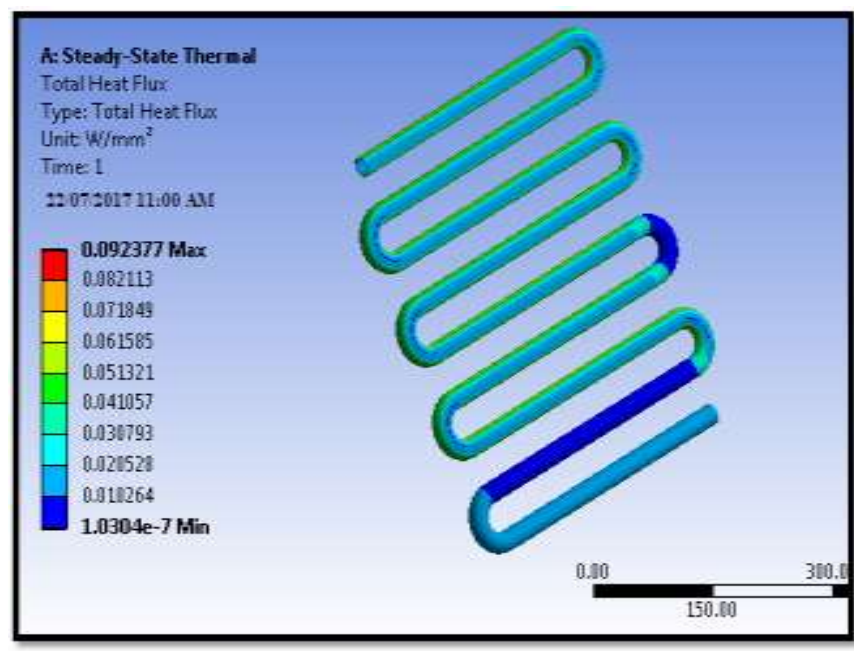

Fig 10: HEAT FLUX

\subsubsection{Condenser Length-405mm}

\section{Material -Aluminum}

\section{Temperature}

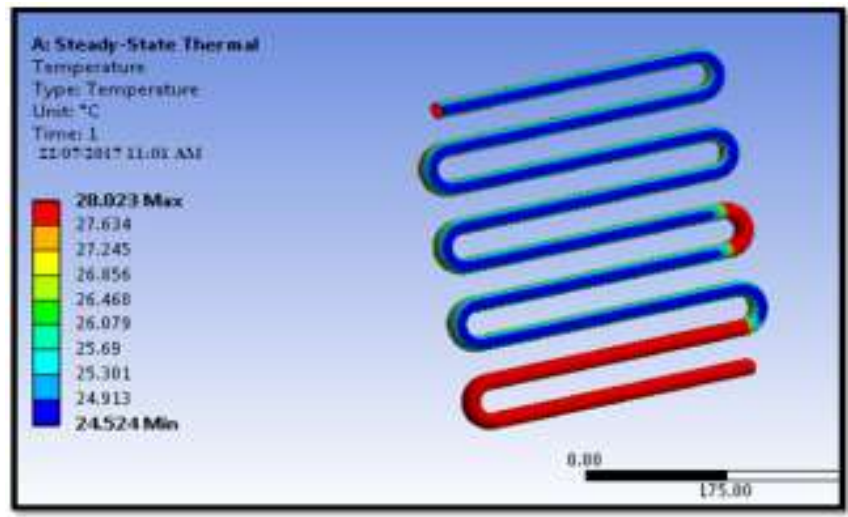

Fig 11: TEMPERATURE

\section{Heat Flux}

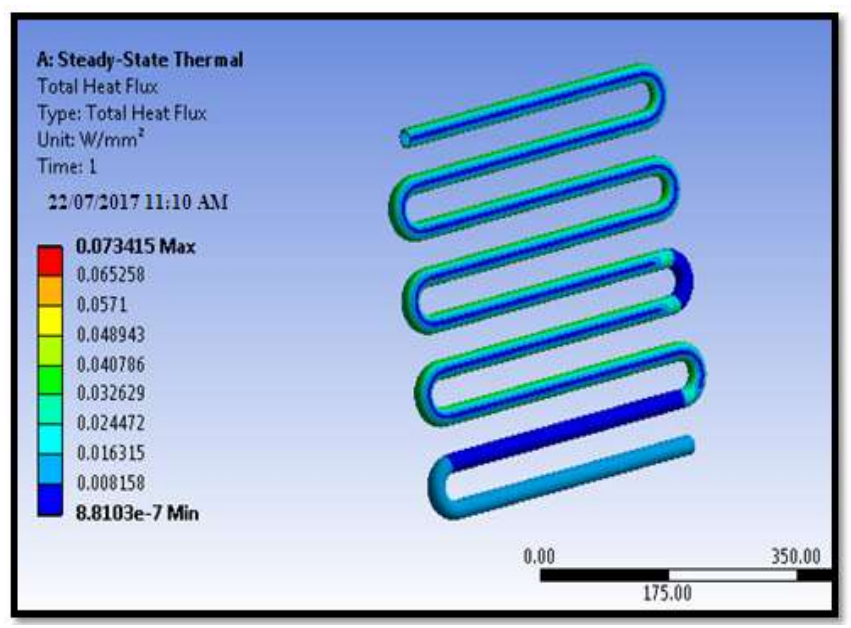

Fig 12: HEAT FLUX

\section{Material -Copper}




\section{Temperature}

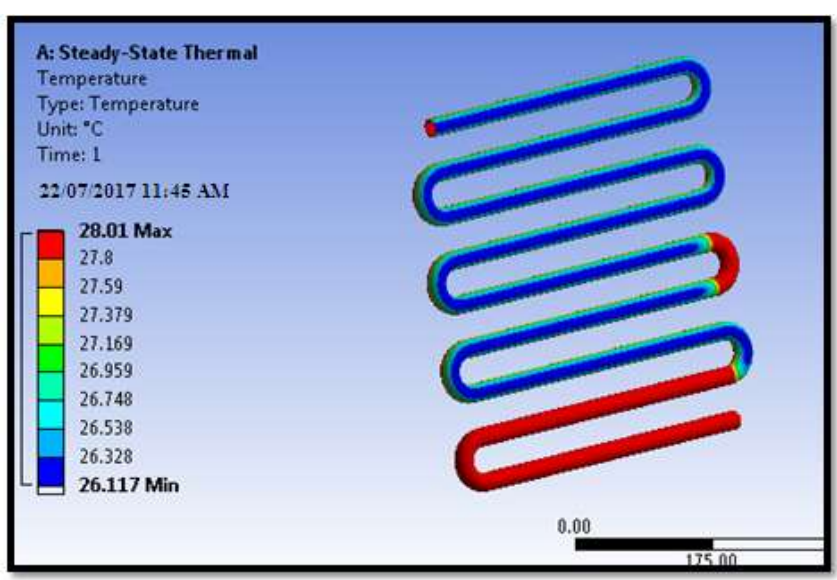

Fig 13: TEMPERATURE

\section{Heat Flux}

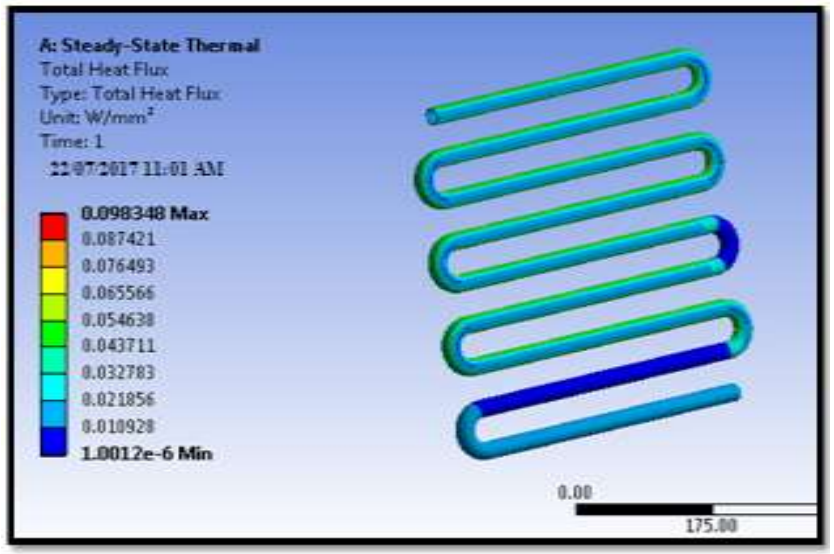

Fig 14: HEAT FLUX

\subsubsection{Condenser Length-465mm}

\section{Material -Aluminum}

\section{Temperature}

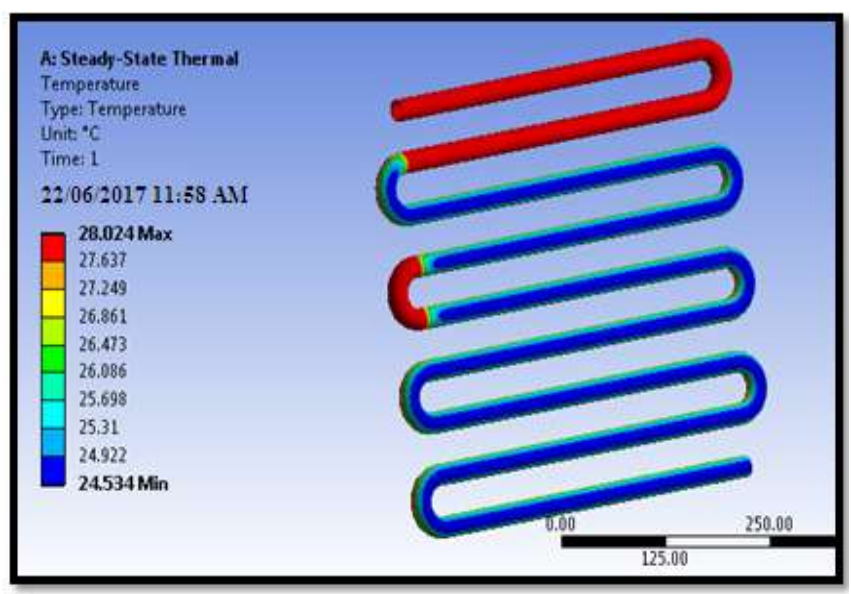

Fig 15: TEMPERATURE

\section{Heat Flux}

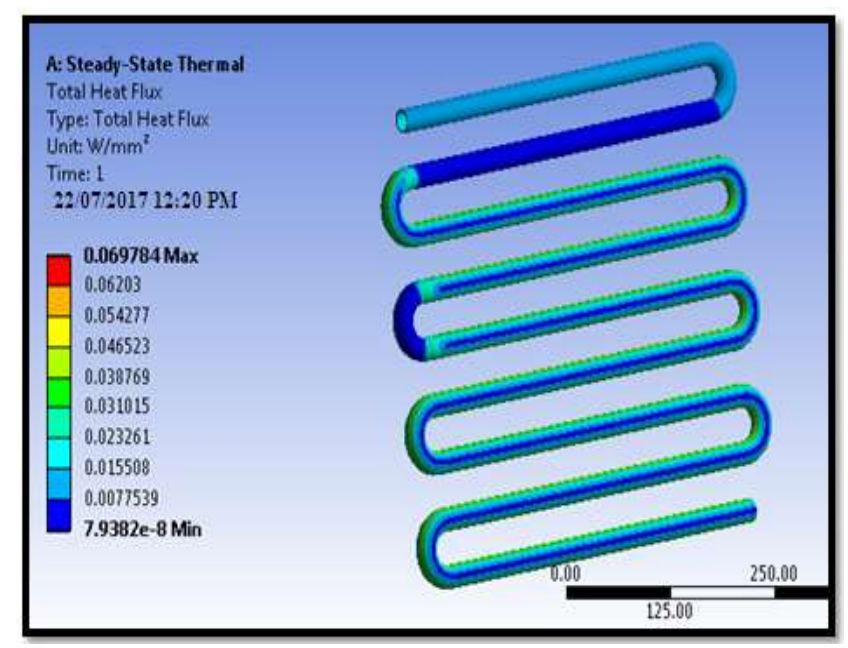

Fig 16: HEAT FLUX

\section{Material -Copper}

\section{Temperature}

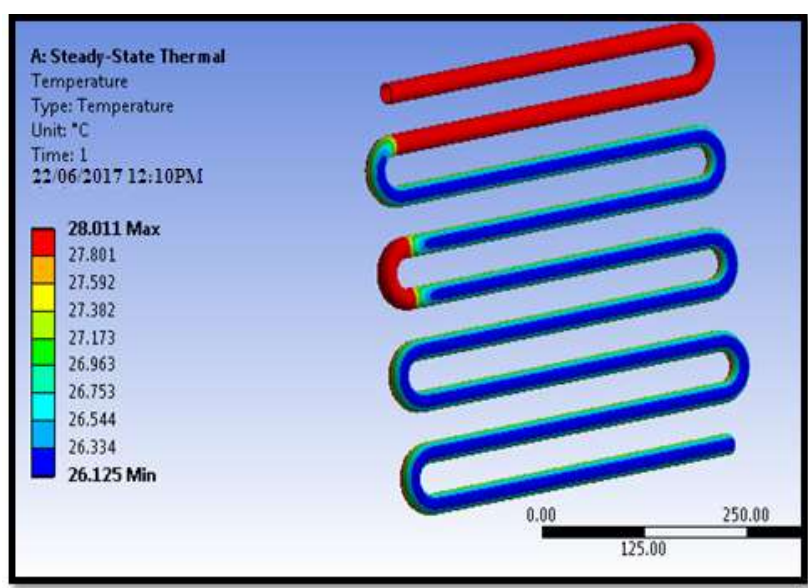

Fig 17: TEMPERATURE

Heat Flux

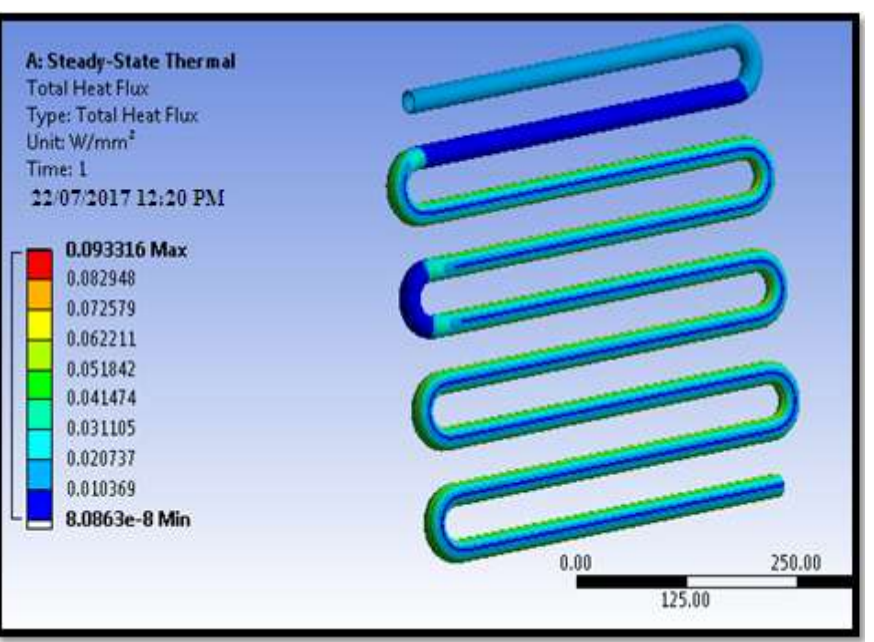

Fig 18: Heat Flux

4.2 CFD Analysis of Condenser 


\subsubsection{At Condenser Length-345mm}

Imported Model

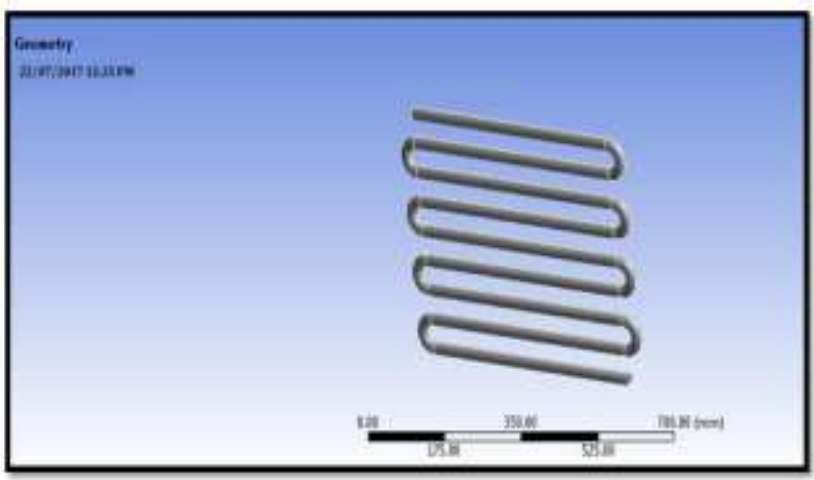

Fig 19: IMPORTED MODEL

\section{MESHED MODEL}

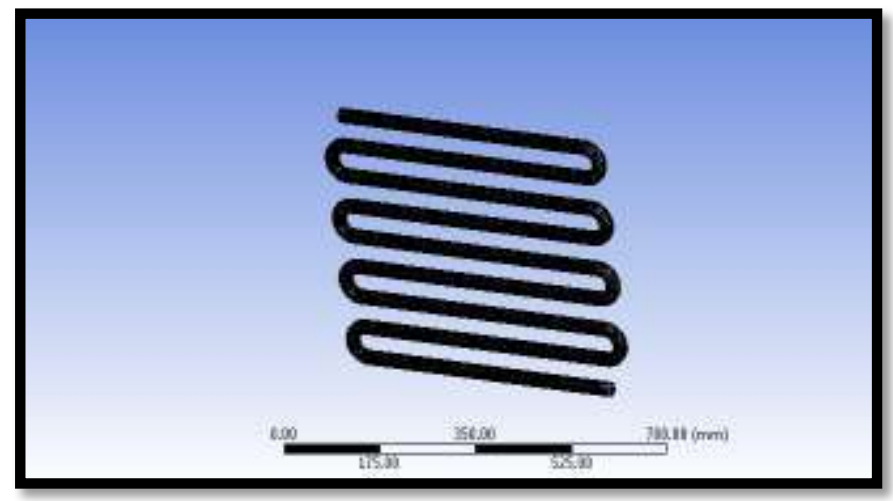

Fig 20: MESHED MODEL

Specifying the Boundaries for Inlet \& Outlet

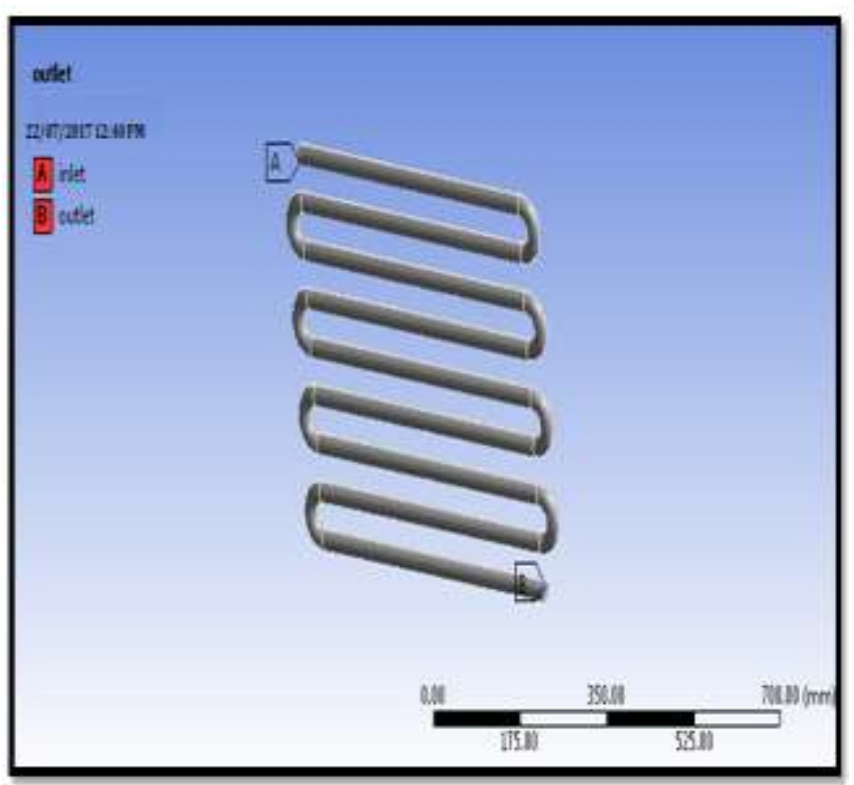

Fig 21: SPECIFYING THE BOUNDARIES FOR INLET \& OUTLET

\section{Pressure}

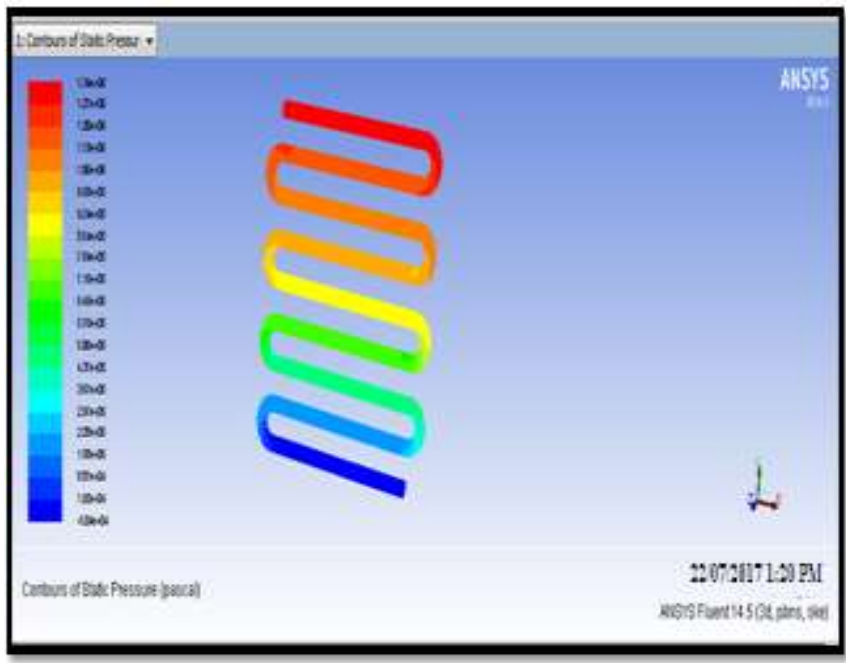

Fig 22: PRESSURE

\section{Temperature}

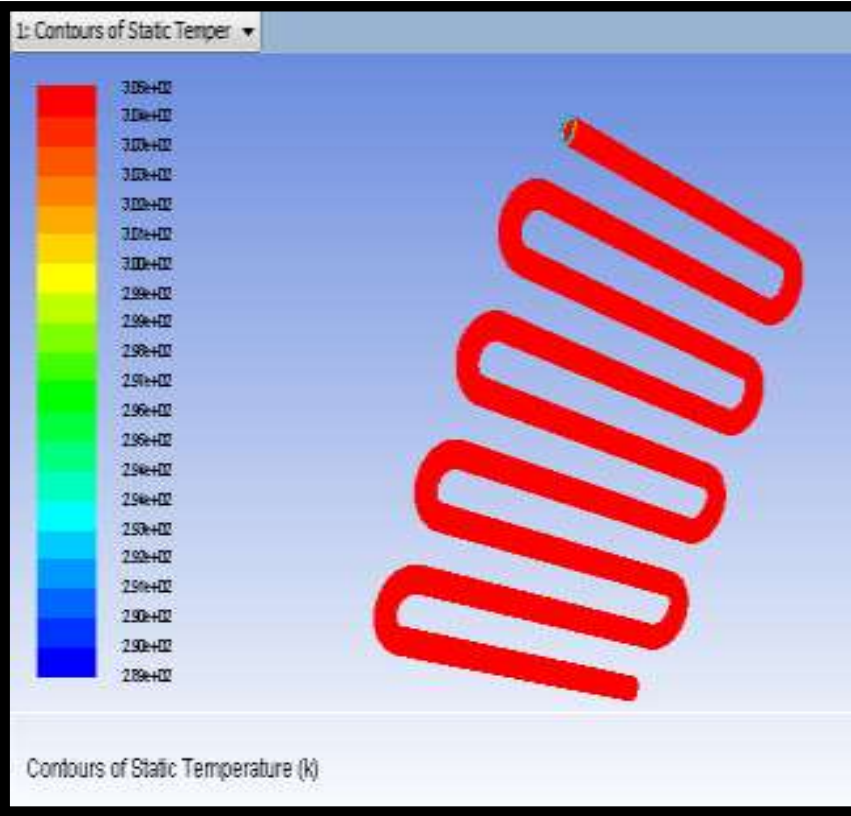

Fig 23: TEMPERATURE

Temperature $=278 \mathrm{~K}$ 


\section{Heat Transfer Coefficient}

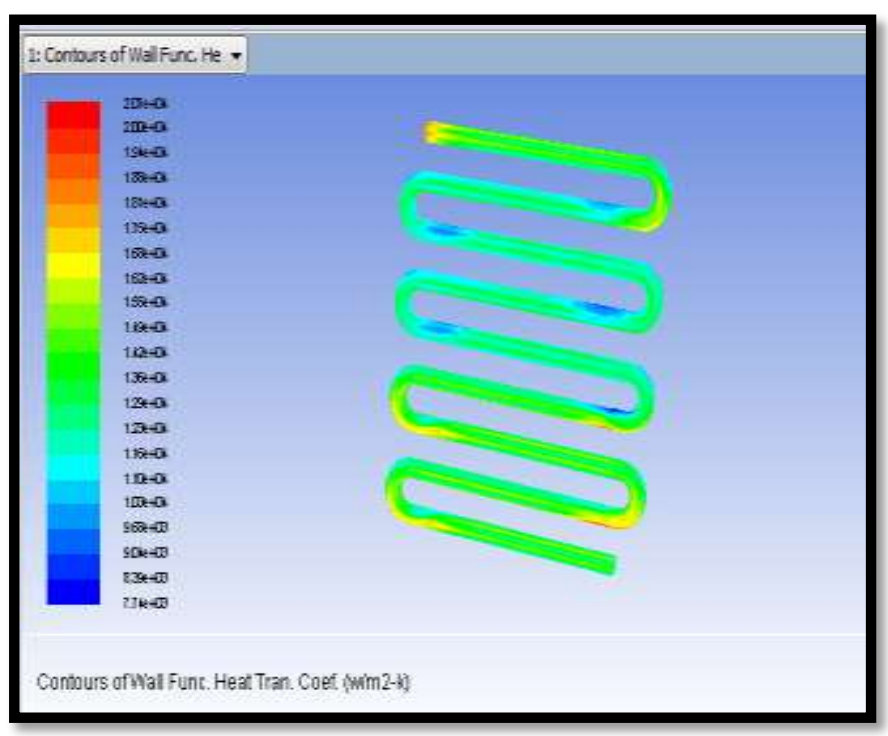

Fig 24: HEAT TRANSFER COEFFICIENT

Mass Flow Rate

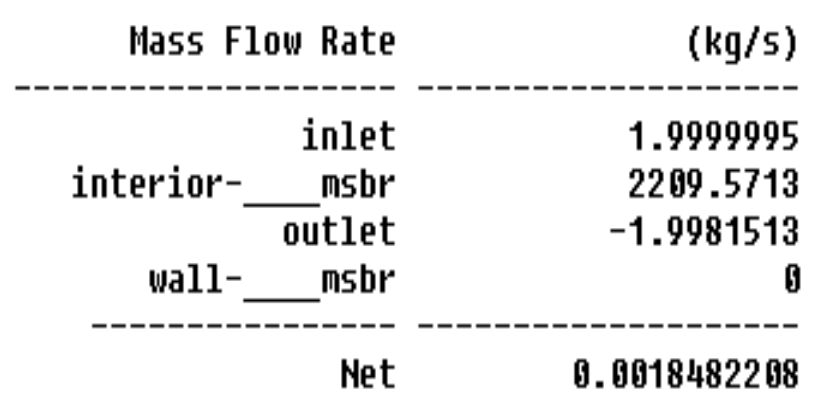

Heat Transfer Rate

Total Heat Transfer Rate

\begin{tabular}{rr}
$\begin{array}{r}\text { inlet } \\
\text { outlet }\end{array}$ & -36353.133 \\
wall- & -11344.112 \\
- & 46663.844 \\
\hline Net & -1033.4014
\end{tabular}

\subsubsection{At Condenser Length-405mm}

\section{Pressure}

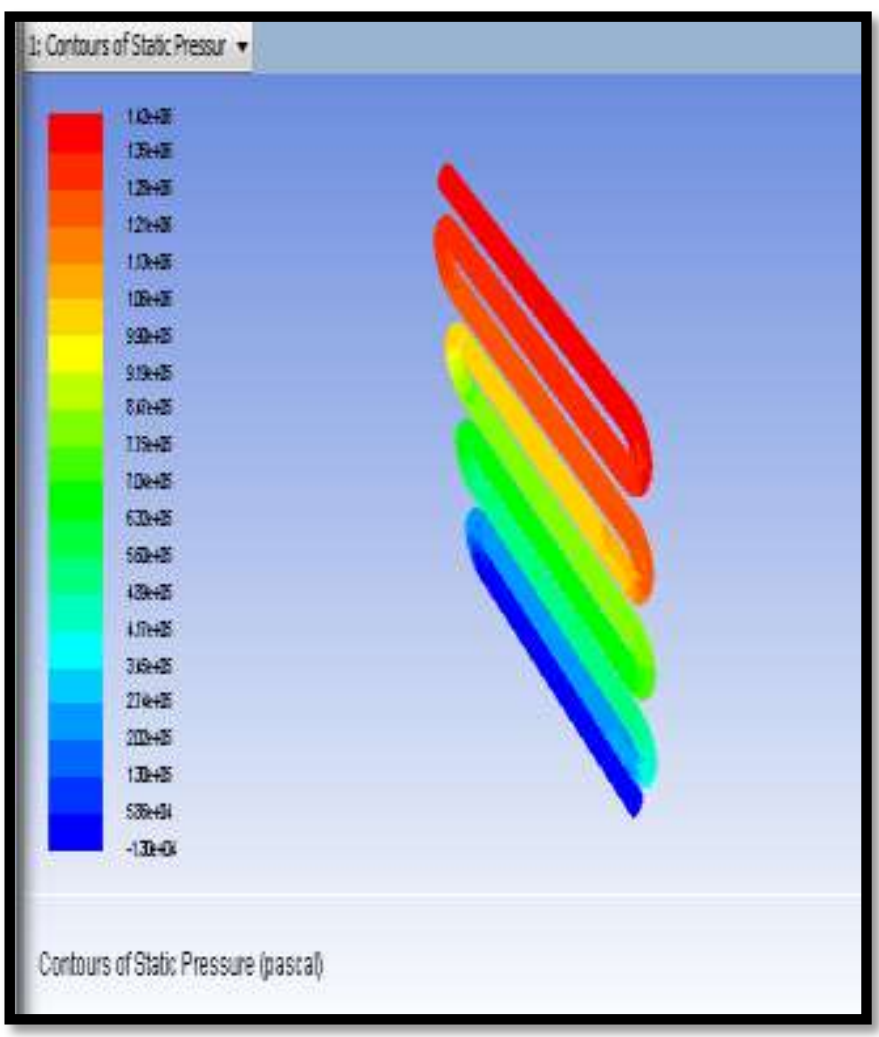

Fig 25: PRESSURE

\section{Temperature}

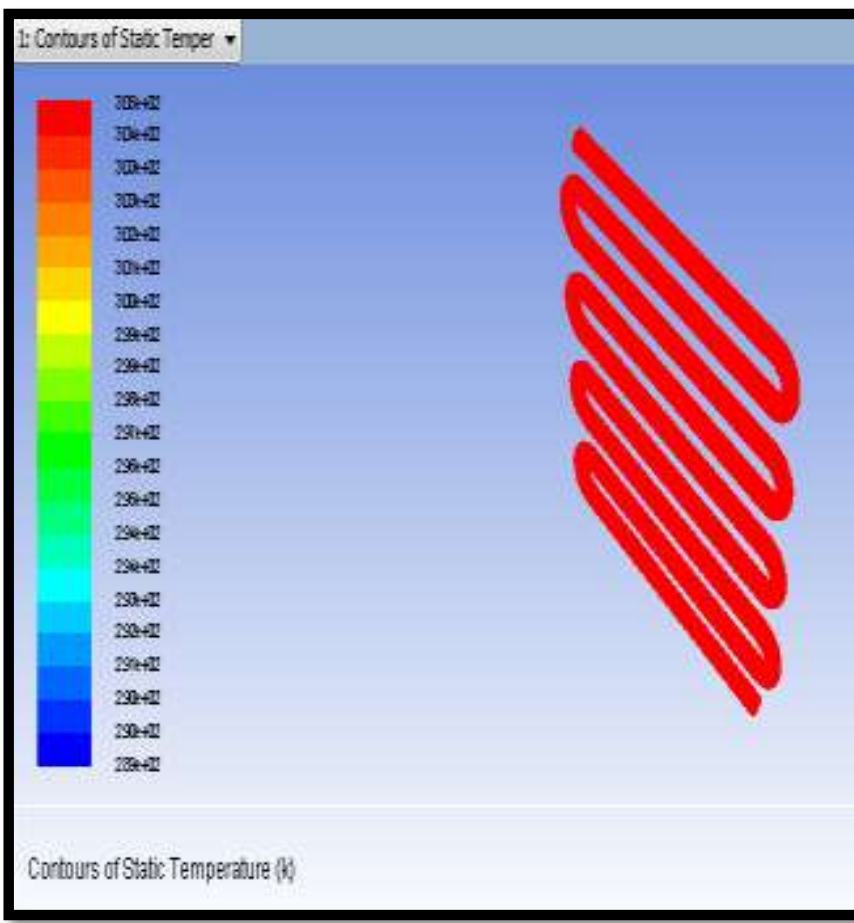

Fig 26: TEMPERATURE 


\section{Heat Transfer Coefficient}

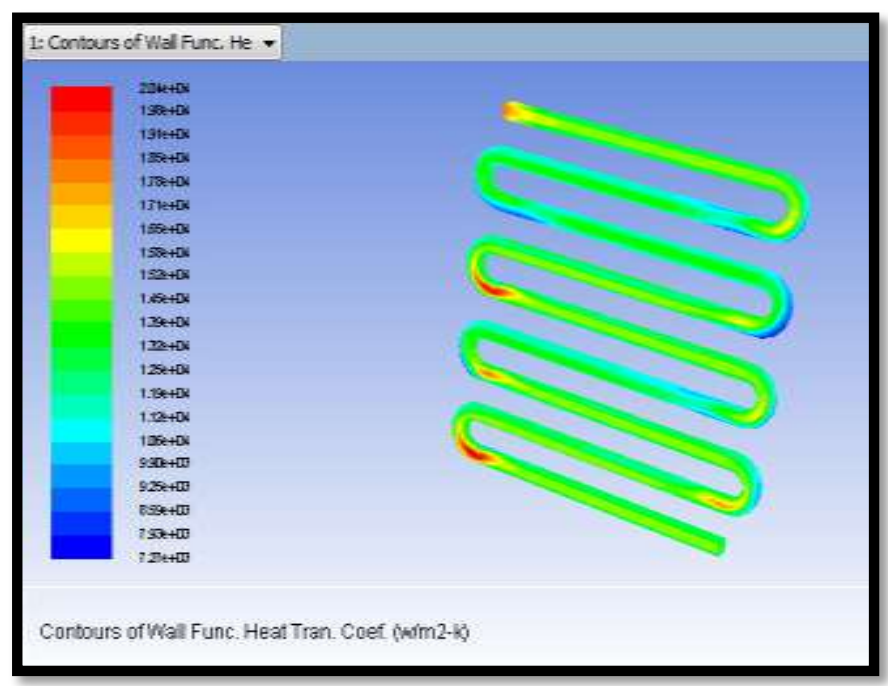

Fig 27: HEAT TRANSFER COEFFICIENT

\section{Mass Flow Rate}

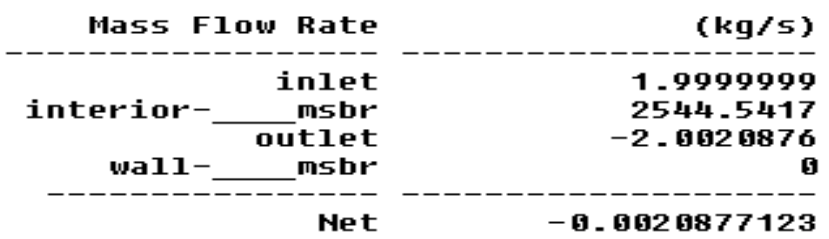

\section{Heat Transfer Rate}

Total Heat Transfer Rate

$\begin{array}{rr}\text { inlet } & -36353.172 \\ \text { outlet } & -11745.454 \\ \text { wall-ris } & 47533.996 \\ - \text { Net } & -564.58958\end{array}$

\subsubsection{At Condenser Length-455mm}

\section{Pressure}

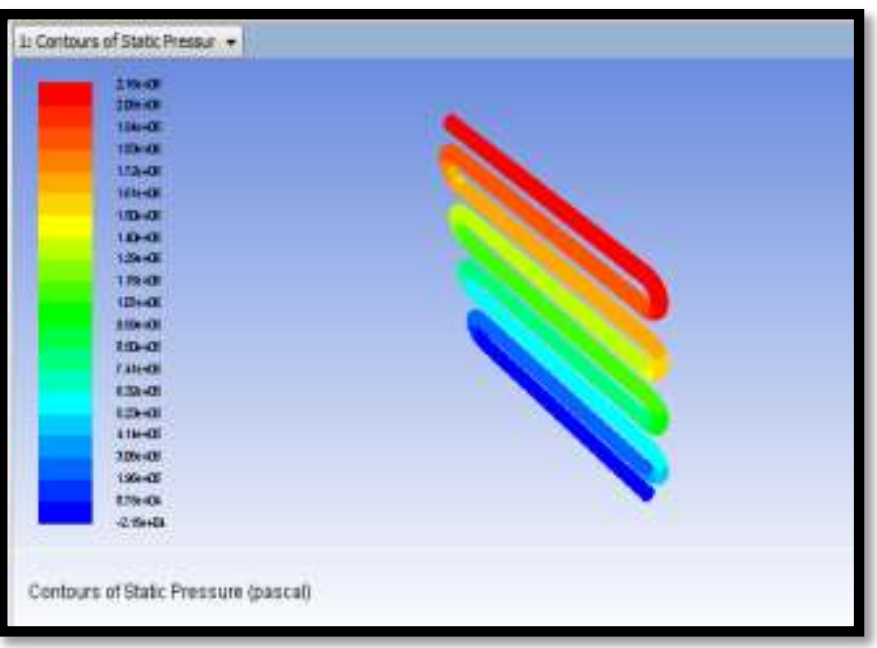

Fig 28: PRESSURE

\section{Temperature}

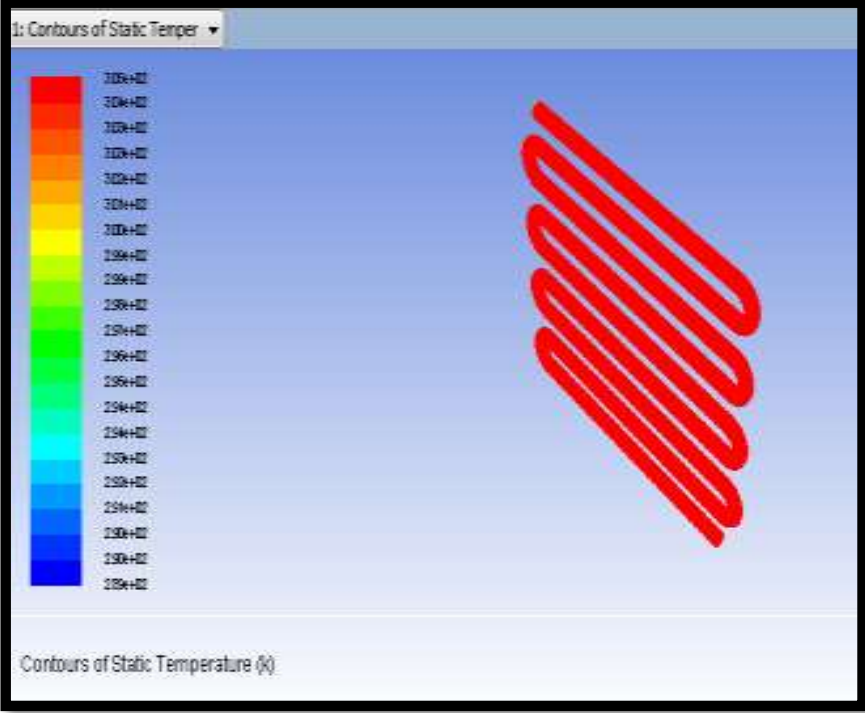

Fig 29: Temperature

\section{Heat Transfer Coefficient}

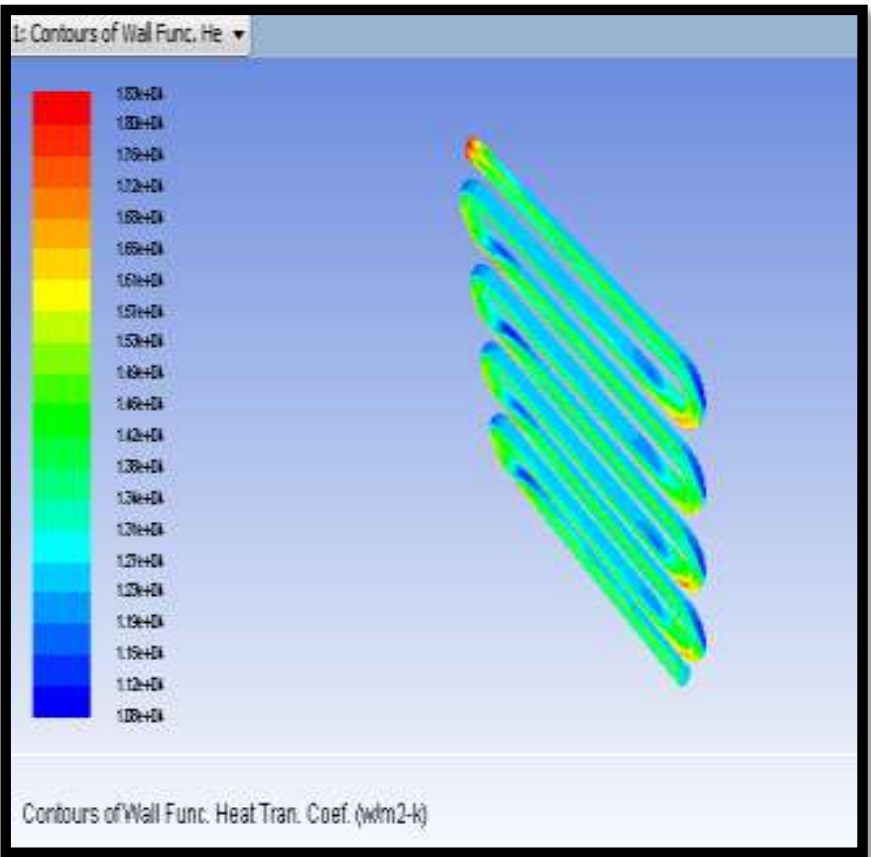

Fig 30: HEAT TRANSFER COEFFICIENT

Mass Flow Rate

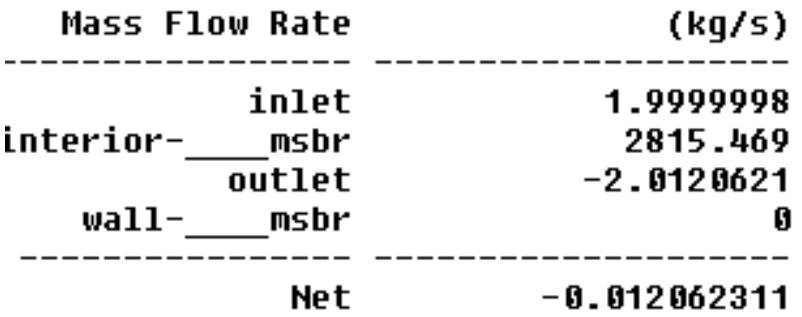




\section{Heat Transfer Rate}

Total Heat Transfer Rate

\begin{tabular}{rr} 
inlet & \\
outlet & -36353.23 \\
wall-robr & -12102.532 \\
\hline--17752.813 \\
Net & -792.9562
\end{tabular}

\subsubsection{At Condenser Length-505mm}

\section{Pressure}

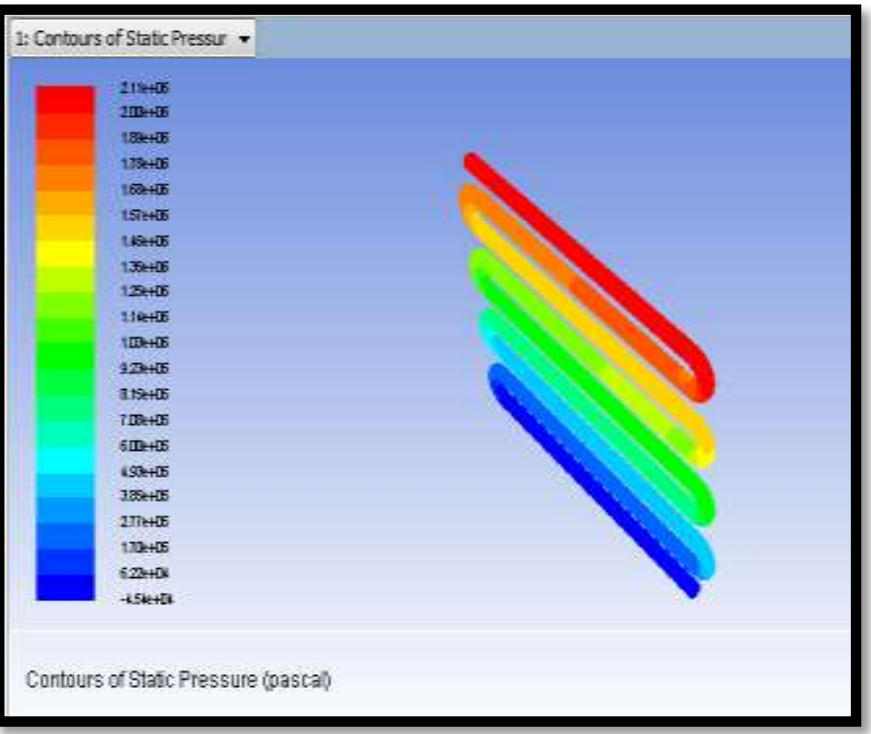

Fig 31: PRESSURE

\section{Temperature}

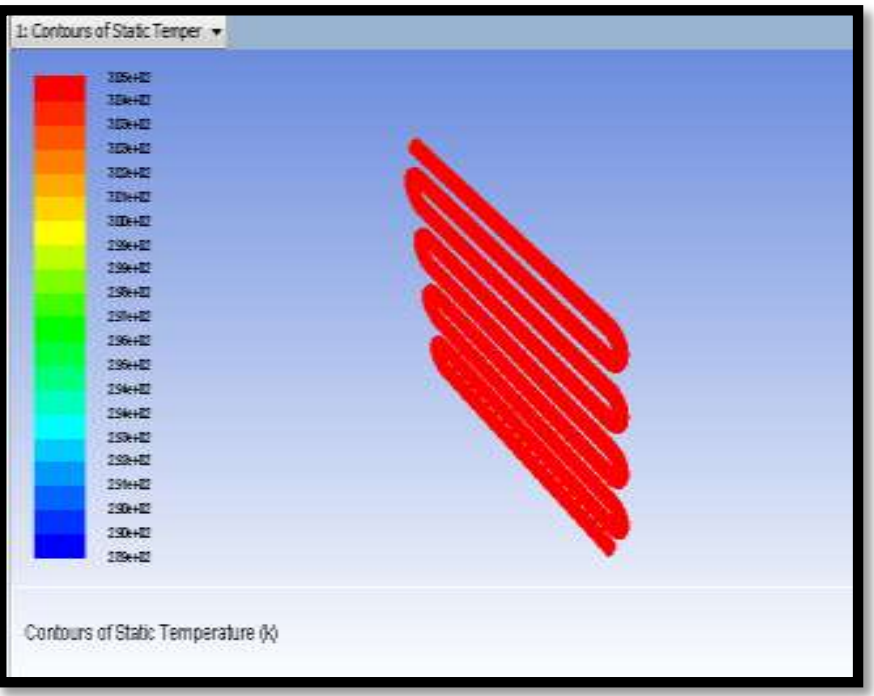

Fig 32: TEMPERATURE

\section{Heat Transfer Coefficient}

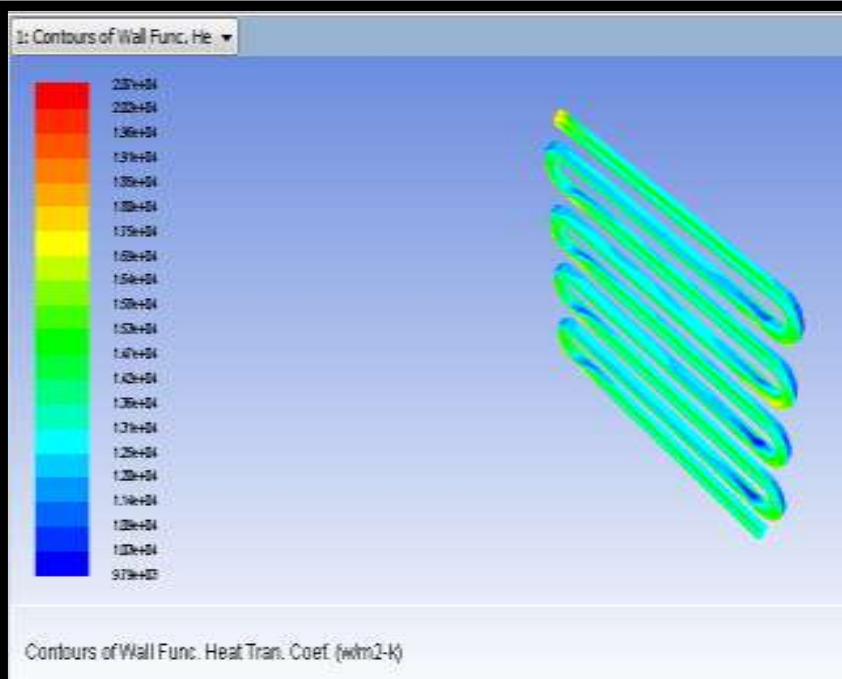

Fig 33: HEAT TRANSFER COEFFICIENT

Mass Flow Rate

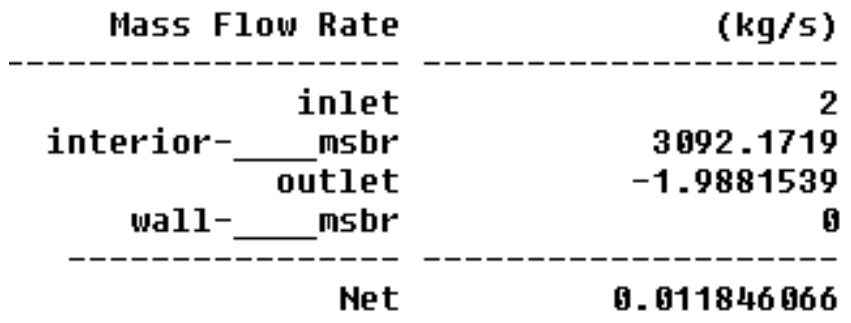

Heat Transfer Rate

Total Heat Transfer Rate (w)

\begin{tabular}{rr} 
inlet & -36353.117 \\
outlet & -12162.339 \\
wall-rmsbr & 48677.563 \\
\hline Net & -377.89355
\end{tabular}

\section{RESULT TABLES}

Table 1: Thermal Analysis

\begin{tabular}{|l|l|l|l|}
\hline Material & $\begin{array}{l}\text { Condenser } \\
\text { length }(\mathbf{m m})\end{array}$ & $\begin{array}{l}\text { Temperature } \\
\text { (k) }\end{array}$ & $\begin{array}{l}\text { Heat } \\
\text { flux } \\
\text { (w/mm }\end{array}$ \\
\hline \multirow{4}{*}{$\begin{array}{l}\text { Aluminum } \\
\text { alloy }\end{array}$} & $\mathbf{3 4 5}$ & 28.026 & 0.068375 \\
\cline { 2 - 4 } & $\mathbf{4 0 5}$ & 28.023 & 0.073415 \\
\cline { 2 - 4 } & $\mathbf{4 6 5}$ & 28.024 & 0.069784 \\
\cline { 2 - 4 } & $\mathbf{5 0 5}$ & 28.025 & 0.069883 \\
\hline \multirow{4}{*}{ Copper } & $\mathbf{3 4 5}$ & 28.012 & 0.092377 \\
\cline { 2 - 4 } & $\mathbf{4 0 5}$ & 28.01 & 0.098348 \\
\cline { 2 - 4 } & $\mathbf{4 6 5}$ & 28.011 & 0.093316 \\
\cline { 2 - 4 } & $\mathbf{5 0 5}$ & 28.015 & 0.093398 \\
\hline
\end{tabular}


TABLE 2: CFD Analysis

\begin{tabular}{|l|l|l|l|l|l|}
\hline $\begin{array}{l}\text { Conde } \\
\text { nser } \\
\text { length } \\
(\mathbf{m m})\end{array}$ & $\begin{array}{l}\text { Pressu } \\
\text { re(Pa) }\end{array}$ & $\begin{array}{l}\text { Tempe } \\
\text { rature } \\
(\mathbf{K})\end{array}$ & $\begin{array}{l}\text { Heat } \\
\text { transfer } \\
\text { coeffiecient } \\
\left(\mathbf{W} /{ }^{\mathbf{m m} 2 \mathbf{k}}\right)\end{array}$ & $\begin{array}{l}\text { Mass } \\
\text { flow } \\
\text { rate(k } \\
\mathbf{g} / \mathbf{s e c})\end{array}$ & $\begin{array}{l}\text { Hea } \\
\mathbf{t} \\
\text { tran } \\
\text { sfer } \\
\text { rate } \\
(\mathbf{w})\end{array}$ \\
\hline $\mathbf{3 4 5}$ & $\begin{array}{l}1.34 \mathrm{e}+ \\
06\end{array}$ & $\begin{array}{l}3.05 \mathrm{e}+ \\
02\end{array}$ & $2.01 \mathrm{e}+04$ & $\begin{array}{l}0.0018 \\
48208\end{array}$ & $\begin{array}{l}103 \\
3.40\end{array}$ \\
\hline $\mathbf{4 0 5}$ & $\begin{array}{l}1.43 \mathrm{e}+ \\
06\end{array}$ & $\begin{array}{l}3.05 \mathrm{e}+ \\
02\end{array}$ & $2.04 \mathrm{e}+04$ & $\begin{array}{l}0.0020 \\
87713\end{array}$ & 564 \\
\hline $\mathbf{4 5 5}$ & $\begin{array}{l}2.15 \mathrm{e}+ \\
06\end{array}$ & $\begin{array}{l}3.05 \mathrm{e}+ \\
02\end{array}$ & $1.83 \mathrm{e}+04$ & 0.0126 & $\begin{array}{l}702 . \\
911\end{array}$ \\
\hline
\end{tabular}

\section{CONCLUSION}

In this thesis heat transfer by convection in refrigeration by varying the condenser length are determined by CFD and thermal analysis. The assessment is out on an air-cooled tube condenser of a vapor compression cycle for refrigeration system.

The materials considered for tubes are Copper and Aluminum alloys. The refrigerants varied will be $\mathrm{R} 12$. CFD analysis is done to determine temperature distribution and heat transfer rates.

In cfd analysis, the heat transfer confident more at condenser length $505 \mathrm{~mm}$.

In thermal analysis, the heat flux is more for copper material at condenser length $405 \mathrm{~mm}$.

So we can conclude that the better material is copper.

\section{REFERENCES}

[1] Air-Conditioning Facts from the 1997 Residential Energy Consumption Survey. http://www.eia.doe.gov/emeu/consumptionbriefs/recs /aircond_use.htm

[2] Hayter, Richard B., Ph.D., P.E. The Future of HVAC: The Perspective of One American. Presented at the 40th anniversary of the Netherlands Technical Association for Building Services (TVVL), June11, 1999, Amsterdam, The Netherlands. http://www.engg.ksu.edu/people/rhayter/tvvlpapr.htm

[3] Home Energy Saver Web page. http://homenergysaver.lbl.gov

[4] Propst, James L. "Air Conditioner Condenser Optimization". Georgia Institute of Technology Thesis. August, 1975.vBeans, E. William. "Computer program for refrigeration cycle analysis".

[5] Thermodynamics and the Design, Analysis, and Improvement of Energy Systems. ASME Adv Energy system Div Publ, AES v27, 1992, ASME, New York, NY, p 153-159. 